\title{
Reassurance on false negatives in the Manchester COVID19 Urgent Eyecare Service (CUES)
}

\author{
Emma Williams ${ }^{1}$, Wendy Craven ${ }^{2}$, Helen Wilson ${ }^{1}$, Felipe Dhawahir-Scala ${ }^{1}$, Matthew Jinkinson ${ }^{2,3}$, William D. Newman ${ }^{1}$ and \\ Robert A. Harper (iD ${ }^{1,4}$
}

(c) The Author(s) 2021

Eye (2022) 36:12-14; https://doi.org/10.1038/s41433-021-01774-w

In June 2020 we described the development and implementation of COVID19 Urgent Eyecare Service (CUES) in Greater Manchester [1]. We subsequently published an evaluation of the service [2], demonstrating a high level of primary care activity, an approximate $\sim 14 \%$ referral rate into secondary care, and reduced footfall into the hospital's emergency eye department (EED). Furthermore, the case mix of patients attending EED appeared to be more complex than that seen prior to introducing CUES, with primary care managing lower risk urgent eyecare presentations; however, one limitation of our evaluation was an acknowledged absence of examination for possible false negatives within CUES. Indeed, in primary eyecare services in general, there has been a paucity of evaluation of the potential for false negatives, although some studies have examined this issue in glaucoma referral filtering services [3-6] and one study did assess clinical safety in a Minor Eye Conditions Service (MECS) [7], a service more closely aligned to CUES in accommodating urgent eyecare in the community. In this article, we present an analysis tracking a large population of cases seen in primary care CUES in Manchester where we specifically monitored the potential for non-referred cases to attend the hospital's EED within 28 days (a proxy for potential missed urgent eyecare cases, i.e. false negatives).

A total of 1027 patient episodes deemed suitable for CUES following initial telephone screening in August, September and October 2020 were subsequently reviewed. Patient episodes rather than patient numbers were considered, since a small proportion of assessments $(N=42)$ were noted to be follow-up assessments carried out for the same patient. The flow diagram in Fig. 1 shows the outcome of the assessments from all episodes. Of 871 episodes resulting in discharge from CUES without referral, a total of 18 patients were identified as having attended Manchester Royal Eye Hospital's (MREH) EED within the following 28 days, giving a potential false-negative rate of $2.07 \%$ (95\% confidence interval $1.23-3.25 \%)$. In order to establish if these cases were genuine false negatives and to examine potential reasons why these patients presented to EED following a CUES discharge, we retrospectively examined the EED records, cross-referencing this information with primary care CUES records (while acknowledging that these provide a summary of optometric consultations in CUES versus any separately held practice practitioner records). This process allowed comparison between diagnostic and management decisions made

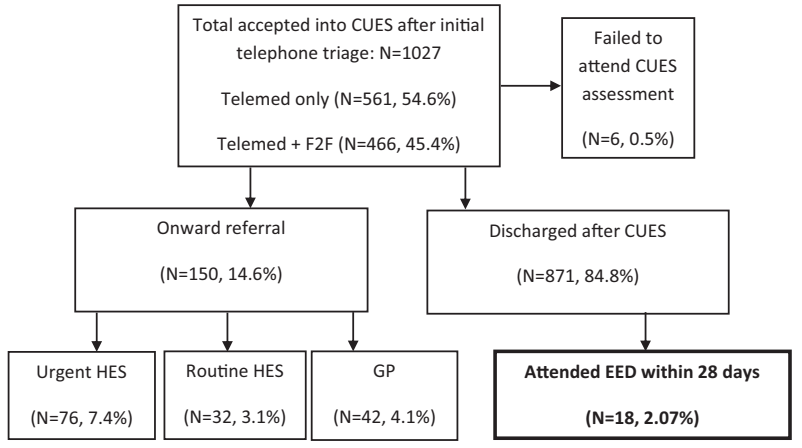

Fig. 1 Flowchart for primary care CUES cases in Manchester and their subsequent management. The proportion of potential falsenegative cases we consider is seen within the context of all nonreferred CUES episodes (871) versus the total number of episodes seen within the service in the evaluation timeline.

in CUES and in EED. The records were reviewed by two EED hospital optometrists (E.W. and H.W.) with EED's consultant head of acute services (F.D.S.) who risk rated each case by consensus into the following categories: A - Agreement on diagnosis/management: No risk to patient; B - Disagreement on diagnosis/management: No/low risk of threat to sight; and C - Disagreement on diagnosis/ management: Moderate/high risk of threat to sight. Table 1 summarises comparative data for CUES and EED diagnoses and management decisions with companion risk ratings.

Reassuringly, in 13 cases there were no concerns over the management decisions taken by the CUES optometrist. In three cases there was disagreement on diagnosis and management, although the disagreement posed a low level of risk to the patient's sight. All three cases were red eye or eye infection dealt with by telemedicine only, following a protocol created for primary care optometrists relevant at the material time, limiting face-to-face assessment of red eye during the pandemic to conditions that were potentially sight-threatening, albeit relying on a high-quality history and symptoms assessment to identify "red flags".

The remaining two cases, in which the disagreement on diagnosis and management was classified as ' $C$ ' (i.e. moderate-to-high risk

\footnotetext{
${ }^{1}$ Manchester Royal Eye Hospital and Manchester Academic Health Sciences Centre, Manchester University NHS Foundation Trust Manchester, Manchester, UK. ${ }^{2}$ Primary Eyecare

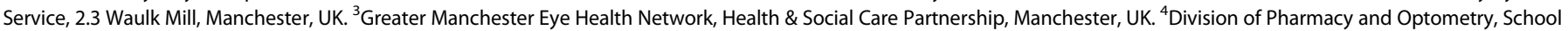
of Health Sciences, Faculty of Biology, Medicine and Health, University of Manchester, Manchester, UK. ${ }^{凶}$ email: robert.harper@mft.nhs.uk
} 


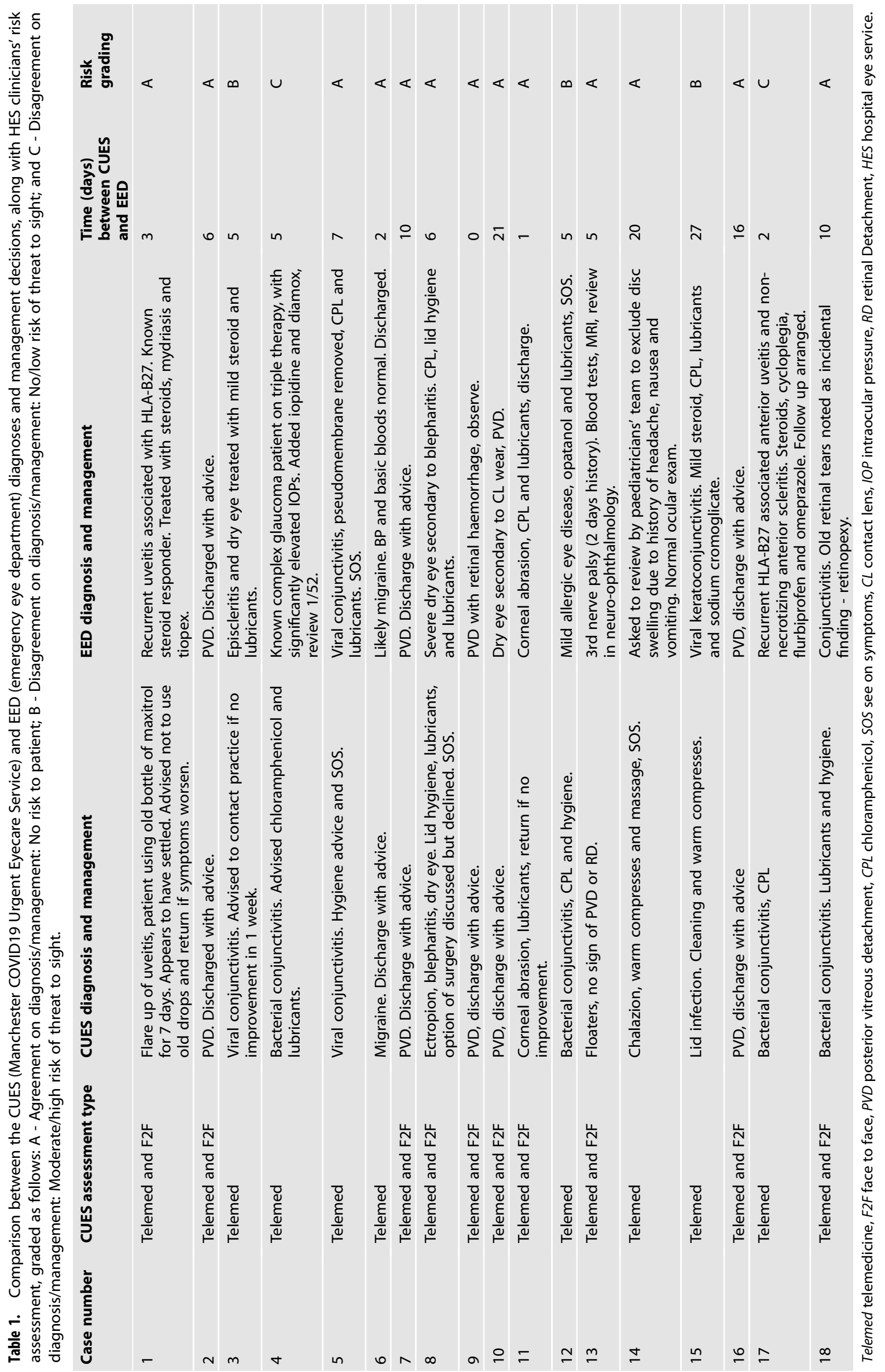


of threat to sight), represented $0.23 \%$ ( $95 \%$ confidence interval $0.03-0.83 \%)$ of all non-referred cases. These cases are considered further: Case 4 (Table 1) involved a 56-year old female patient assessed by telemedicine, noted to have a red right eye with sticky discharge. The vision in the right eye was noted to be worse and the left was stable. After CUES assessment the patients was diagnosed with bacterial conjunctivitis and treatment with chloramphenicol and lubricants was advised. Although the patient subsequently reattended CUES for a face-to-face assessment and was referred, her referral was routine, and she attended EED herself complaining of a "pressure sensation" within the eyes and was found to have intra-ocular pressures of $31 \mathrm{mmHg}$ bilaterally with very narrow angles due to bilateral peripheral anterior synechiae. The patient had a history of poorly controlled narrow angle glaucoma, treated with peripheral iridotomies, bilateral cataract surgery, and triple topical glaucoma therapy. Previously, following her right cataract procedure in 2019, she had attended EED for emergency treatment. Since her more recent EED episode post-CUES, she has undergone glaucoma surgery. In CUES, her complex glaucoma history did not appear to have been elucidated. Case 17 (Table 1) involved a 45-year old female diagnosed with bacterial conjunctivitis in CUES and treated with chloramphenicol for a red, painful, watery left eye. The patient attended EED 2 days later, where a history of recurrent acute anterior uveitis in association with ankylosing spondylitis was noted at triage. She was found to have a recurrence of uveitis with non-necrotizing anterior scleritis and was treated accordingly. A full history and symptoms assessment, including previous eye problems and general health status, would have alerted the CUES practitioner to the more likely diagnosis of uveitis.

In conclusion, this evaluation of a non-referred population seen in primary care CUES supports the view that the service is clinically safe. The false-negative rate of $0.23 \%$ for moderate-to-high risk of sight loss cases in the cohort reviewed is reassuringly low within the context of: first, the pandemic and emphasis on telemedicine at the material time; second, CUES evolving to accommodate a higher proportion of face-to-face assessments and with the potential for further guidance for participating optometrists about the importance of a thorough history and symptoms evaluation; and finally, CUES comparing favourably with currently commissioned primary care services where false-negative rate evidence is available [3-7]. We believe that this additional analysis and our earlier evaluation strongly supports the ongoing commissioning of CUES in primary care.

\section{REFERENCES}

1. Harper RA, Dhawahir-Scala F, Wilson H, Gunn PJG, Jinkinson M, Pretty IA. et al. Development and implementation of a Greater Manchester COVID19 Urgent Eyecare Service. Eye (Lond). 2021;35:705-8. https://doi.org/10.1038/s41433-0201042-6.

2. Kanabar R, Craven W, Wilson H, Rietdyke R, Dhawahir-Scala F, Jinkinson M. et al. Evaluation of the Manchester COVID-19 Urgent Eyecare Service (CUES). Eye. 2021; https://doi.org/10.1038/s41433-021-01522-0.

3. Devarajan N, Williams GS, Hopes M, O'Sullivan D, Jones D. The Carmarthenshire Glaucoma Referral Refinement Scheme, a safe and efficient screening service. Eye (Lond). 2011;25:43-9.
4. Ratnarajan G, Kean J, French K, Parker M, Bourne R. The false negative rate and the role for virtual review in a nationally evaluated glaucoma referral refinement scheme. Ophthalmic Physiol Opt. 2015;35:577-81.

5. Gunn PJG, Marks JR, Konstantakopoulou E, Edgar DF, Lawrenson JG, Roberts SA. et al. Clinical effectiveness of the Manchester Glaucoma Enhanced Referral Scheme. Br J Ophthalmol. 2019;103:1066-71.

6. Harper RA, Gunn PJG, Fenerty CH, Spry PGD, Lawrenson JG. Care pathways for glaucoma detection and monitoring in the UK. Eye. 2020;34:89-102.

7. Konstantakopoulou E, Harper RA, Edgar DF, Larkin G, Janikoun S, Lawrenson JG. Clinical safety of a minor eye conditions scheme in England delivered by community optometrists. BMJ Open Ophthalmol. 2018;3:e000125. https://doi.org/ 10.1136/bmjophth-2017-000125.

\section{ACKNOWLEDGEMENTS}

The authors are grateful to the following: Alistair Rutherford (Performance and Quality Improvement Manager at Manchester Health and Care Commissioning and Manchester (CG) for facilitating access to details of EED attendances from primary care CUES cases; and Mairead Gallagher (clerical team) at MREH for assisting with retrieval of clinical records within MREH.

\section{AUTHOR CONTRIBUTIONS}

R.A.H., W.C. and H.W. conceived and designed the study, supported by M.J., F.D.S. and W.D.N.; E.W., W.C. and H.W. performed the data collection; E.W. and H.W. performed the analysis, supported by F.D.S. and W.C.; E.W. and R.A.H. wrote the manuscript; W.C., H.W., F.D.S., M.J. and W.D.N. provided critical review of the manuscript.

\section{COMPETING INTERESTS}

M.J. is Clinical Director and W.C. is Clinical Governance Lead at Primary Eyecare Services, holder of the contract with Manchester CCG in relation to CUES and other primary care optometry delivered services.

\section{ADDITIONAL INFORMATION}

Correspondence and requests for materials should be addressed to Robert A. Harper.

Reprints and permission information is available at http://www.nature.com/ reprints

Publisher's note Springer Nature remains neutral with regard to jurisdictional claims in published maps and institutional affiliations.

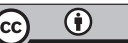

Open Access This article is licensed under a Creative Commons Attribution 4.0 International License, which permits use, sharing, adaptation, distribution and reproduction in any medium or format, as long as you give appropriate credit to the original author(s) and the source, provide a link to the Creative Commons license, and indicate if changes were made. The images or other third party material in this article are included in the article's Creative Commons license, unless indicated otherwise in a credit line to the material. If material is not included in the article's Creative Commons license and your intended use is not permitted by statutory regulation or exceeds the permitted use, you will need to obtain permission directly from the copyright holder. To view a copy of this license, visit http://creativecommons. org/licenses/by/4.0/.

(c) The Author(s) 2021 